PAPER

\title{
Survival and quality of life outcome after mechanical ventilation in elderly stroke patients
}

\author{
C Foerch, K R Kessler, D A Steckel, H Steinmetz, M Sitzer
}

See end of article for authors' affiliations

.....................

Correspondence to: Dr C Foerch, Department

of Neurology, Johann Wolfgang GoetheUniversity, Schleusenweg 2-16, 60590 Frankfurt am Main, Germany; foerch@ em.uni-frankfurt.de

Received 20 June 2003

In revised form

6 November 2003

Accepted

6 November 2003
J Neurol Neurosurg Psychiatry 2004;75:988-993. doi: 10.1136/jnnp.2003.021014

Objectives: Mortality is high and functional outcome poor in mechanically ventilated stroke patients. In addition, age $>65$ years is an independent predictor of death at 2 months among these patients. Our objective was to determine survival rates, functional outcome, and quality of life (QoL) in stroke patients older than 65 years requiring mechanical ventilation.

Methods: A prospective cohort study with an additional cross-sectional survey in 65 patients aged 65 years and older (mean age (SD): 75.6 (6.0) years) with ischaemic or haemorrhagic stroke who underwent mechanical ventilation. Main outcome measures were survival rate at 6 months, and Barthel Index (BI), modified Rankin Scale, and QoL at 15.8 (SD 8.0) months.

Results: Survival rate at 6 months was $40 \%$. Elective intubation (odds ratio (OR) 13.6; $p=0.002$ ) was the only independent positive predictor for survival, while age $>77.5$ years (OR $0.1 ; p=0.004$ ) and white blood count $>10 / \mathrm{nl}$ at admission (OR $0.31 ; \mathrm{p}=0.032$ ) were independent negative predictors for survival at 6 months. At the time of the cross-sectional survey, Bl was $>70$ in five out of 22 patients, 35-70 in three and $<35$ in the remaining 14 patients. QoL was impaired primarily in the physical domain, whereas the psychosocial domain was less affected.

Conclusions: Although only $40 \%$ of elderly patients intubated in the acute phase of stroke survived at least 6 months, one in four survivors recovered to a good functional outcome with a reasonable QoL. Elderly stroke patients need to be selected carefully for intensive care treatment, but elective intubation to allow diagnostic procedures should not be withheld primarily based on their age.
$\mathrm{P}$ revious studies showed that severe ischaemic or haemorrhagic stroke requiring intensive care therapy and mechanical ventilation (MV) is associated with a poor prognosis. Approximately $60 \%$ of the patients die within the first 2 months and the majority of the survivors remain severely disabled. ${ }^{1-4}$ Age $>65$ years was identified as an independent predictor of death at 2 months in mechanically ventilated stroke patients, ${ }^{4}$ and according to recent population based data, $75-85 \%$ of stroke patients fall within this age range. ${ }^{56}$ The overall 28 day mortality of stroke is approximately $25 \%$, and $10 \%$ of all stroke patients require MV during the acute phase of stroke therapy. ${ }^{78}$ Thus, the question of whether intensive care therapy should be minimised in elderly stroke patients is highly relevant for stroke neurologists and intensivists considering the limited intensive care capacities.

The principal dilemma is that withholding MV will lead to a higher early mortality, whereas intensive care therapy probably improves survival at the cost of severe neurological deficits; reliable early predictors of functional outcome are lacking. On the other hand, new diagnostic and therapeutic strategies (for example, acute stroke MRI, thrombolysis, or mild hypothermia) may improve prognosis and outcome, but often require at least temporary airway protection and mechanical ventilation.

This prospective study of elderly stroke patients (aged 65 years and older) with severe ischaemic or haemorrhagic stroke requiring $\mathrm{MV}$ in the acute phase focused particularly on the impact of the combined risks of intensive care therapy and mechanical ventilation together with higher age on survival and quality of life (QoL) outcome.

\section{METHODS}

\section{Sample}

Between October 1998 and January 2001, 65 consecutive acute stroke patients aged 65 years and older who required
MV within $48 \mathrm{~h}$ of symptom onset were prospectively included in this single centre analysis. Diagnosis of cerebral ischaemia or intracerebral haemorrhage was confirmed in all cases. Patients were followed using a prospective hospitalbased anonymous stroke registry containing demographic, prehospital, clinical, and procedural data. ${ }^{9}$ All patients registered in this database were contacted for a follow up survey 6 months after admission in written form using a questionnaire to determine survival and status of dependency.

In addition, we performed a cross-sectional survey of the surviving patients with a mean follow up time of 15.8 (SD 8 ) months (range: 9-33 months) to determine functional outcome and QoL using the three instruments described below. All patients who were still alive at the 6 month follow up were contacted by telephone using a standardised interview and in written form using questionnaires including information on whether the patients filled out the questionnaires by themselves, or with the help of a proxy. Written informed consent for participation in the cross-sectional survey was obtained from all patients or their next of kin.

\section{Procedures}

In addition to the data recorded in the aforementioned stroke registry, more detailed information on the initial neurological deficit (for example, anisocoria, pupillary reflexes, forced gaze deviation, corneal reflexes, Babinski's sign) were prospectively collected. From the laboratory analysis performed upon admission, white blood count (WBC, in cells/nl) and the serum concentrations of C-reactive protein $(\mathrm{mg} / \mathrm{dl})$ and glucose $(\mathrm{mg} / \mathrm{dl})$ were obtained.

Abbreviations: $\mathrm{BI}$, Barthel Index; $\mathrm{Cl}$, confidence interval; $\mathrm{MCA}$, middle cerebral artery; mRS, modified Rankin Scale; MV, mechanical ventilation; OR, odds ratio; QoL, quality of life; SIP, sickness impact profile; WBC, white blood count 
Furthermore, time and date of intubation, tracheostomy (if performed), and extubation were recorded and the reasons for intubation were classified as follows: (a) "neurological", if the patient was intubated primarily because of neurological deterioration, coma $(\mathrm{GCS}<9)$, or loss of protective reflexes; (b) "respiratory", if the patient had primary respiratory failure defined as arterial $\mathrm{Po}_{2}<8 \mathrm{kPa}(60 \mathrm{~mm} \mathrm{Hg})$ and/or arterial $\mathrm{PCO}_{2}>8 \mathrm{kPa}$ and/or breathing rate $>40 / \mathrm{min}$ and/or arterial $\mathrm{pH}<7.3$ (for example, due to aspiration or pulmonary oedema); and (c) "elective", if the patient had to be intubated in order to carry out a diagnostic procedure or intervention (magnetic resonance imaging, angiography, surgical intervention), which would have been impossible otherwise (for example, in uncooperative patients). End of MV was defined as extubation, death or, if tracheostomy was performed, continuous sufficient ventilation via the tracheostomy tube without need of mechanical support for more than $24 \mathrm{~h}$.

All patients were treated according to current standards in stroke management. ${ }^{10}$ Ten patients fulfilled the criteria for intravenous thrombolytic therapy using rt-PA $(0.9 \mathrm{mg} / \mathrm{kg})$. Another 10 patients who presented within $5 \mathrm{~h}$ following symptom onset and had MR angiography proven occlusion of the middle cerebral artery (MCA) received intra-arterial thrombolysis with urokinase (1-1.5 Mio. U.). In three patients with complete MCA infarction and impending herniation, decompressive hemicraniectomy was performed. An external ventricular drainage was placed in three patients with intracranial haemorrhage, and in two patients intracerebral haematoma was evacuated.

\section{Instruments}

In the cross-sectional survey, we determined Barthel Index (BI), modified Rankin Scale (mRS), and the Stroke Adapted Sickness Impact Profile (SA-SIP30) in its German version. ${ }^{11}$ Additional data on current living situation were obtained, and the interviewer asked the patient or caregiver, if they in retrospect would again decide in favour of MV considering the actual outcome.

The original BI is a 10-item disability score with scores ranging between 0 (highest dependency) and 100 (lowest dependency) with 5-point increments. ${ }^{12}$ Outcome was categorised primarily based on the BI (good: 75-100; moderate: 35-70; poor: $0-30$ ). The $\mathrm{mRS}$ is a widely used handicap rating scale ranging from 0 (full independency) to 5 points (full dependency). ${ }^{13}$

The Sickness Impact Profile (SIP) in its original version consists of 136 dichotomous items grouped into 12 categories and is a frequently used generic tool to measure healthrelated QoL. ${ }^{14}$ From it, the more stroke-specific SA-SIP30 was derived and validated, and comprises of 30 items in six categories and two dimensions (physical and psychosocial). ${ }^{15}$ The scores range from 0 to $100 \%$ of maximal dysfunction.

\section{Statistics}

All statistical analyses were performed using a commercial software package (SPSS 10.0, SPSS Inc., Chicago, IL, USA). Survival curves were obtained by the Kaplan-Meier method. Univariate association was analysed using $\chi^{2}$ tests. Independent predictors of survival at 6 months were identified by logistic regression analysis. $\mathrm{p} \leqslant 0.05$ was considered as significant. All statistical tests were performed two-tailed.

To further analyse the influence of age, patients were dichotomised by using the average life expectancy at birth calculated to be 77.5 years for the year 2000 in Germany. ${ }^{16}$

\section{RESULTS}

A total of 65 patients (30 female) aged 75.6 (SD 6.0) years (range: 65.4-89.2 years) were included in the analysis. As is summarised in table 1, 46 patients presented with ischaemic stroke, which was related to the anterior circulation in 35 and to the posterior circulation in 11 patients. Due to our selection of stroke patients requiring MV, 28 patients had infarcts involving at least $50 \%$ of the MCA territory. In 19 patients an intracerebral haemorrhage was diagnosed, which was supratentorial in 15 and infratentorial in four patients. Haematoma volumes ranged between 4 and $140 \mathrm{ml}$.

Prior to their stroke, 50 patients had a BI of 100 points and only four patients had a $\mathrm{BI}<60$ points indicating that the majority of patients had been in a good to excellent functional state.

Patients were admitted to our department on average 8.6 (14.8) $\mathrm{h}$ following the onset of clinical symptoms, but most patients $(n=43)$ were admitted within the first $6 \mathrm{~h}$ after symptom onset. A total of 49 patients were intubated within $24 \mathrm{~h}$ after symptom onset, 19 of whom were already intubated at hospital admission.

The majority of patients $(n=45 ; 69 \%)$ were intubated for neurological reasons, seven patients $(11 \%)$ for respiratory reasons, and 13 patients $(20 \%)$ electively, that is, primarily to perform diagnostic or therapeutic procedures. The duration of MV was not significantly different between these three indication groups (for "neurological" 7.3 (SD 5.5), for "respiratory" 5.8 (6.1), and for "elective" 7.0 (7.3) days).

Average length of hospital stay was 16 (14.7) days and ranged from 1 to 81 days. At hospital discharge, 39 patients $(60 \%)$ were still alive. Causes of in-hospital deaths were massive brain oedema with subsequent herniation $(n=21$ patients), sepsis with multiple organ failure $(n=2)$, sudden cardiac arrest $(n=1)$, and pulmonary embolism $(n=2)$.

Of the surviving 39 patients, 30 were transferred to rehabilitation institutions, seven to primary care hospitals, one directly to a nursing home, and one was discharged home.

\section{Results of the 6 month follow up}

At the 6 month follow up, 26 stroke patients (18 ischaemic, eight haemorrhagic) were still alive, indicating a survival rate of $40 \%$. Table 1 shows that survival was related to the size of infarction or haemorrhage. The lowest survival rate was seen in patients with total MCA or posterior circulation infarction, or with haemorrhage volumes $>50 \mathrm{ml}$.

The Kaplan-Meier survival curve (fig 1) shows that 29 of the 39 patients (74\%) who did not survive the first 6 months following their stroke, died in the acute and immediate subacute phase, that is within the first 30 days.

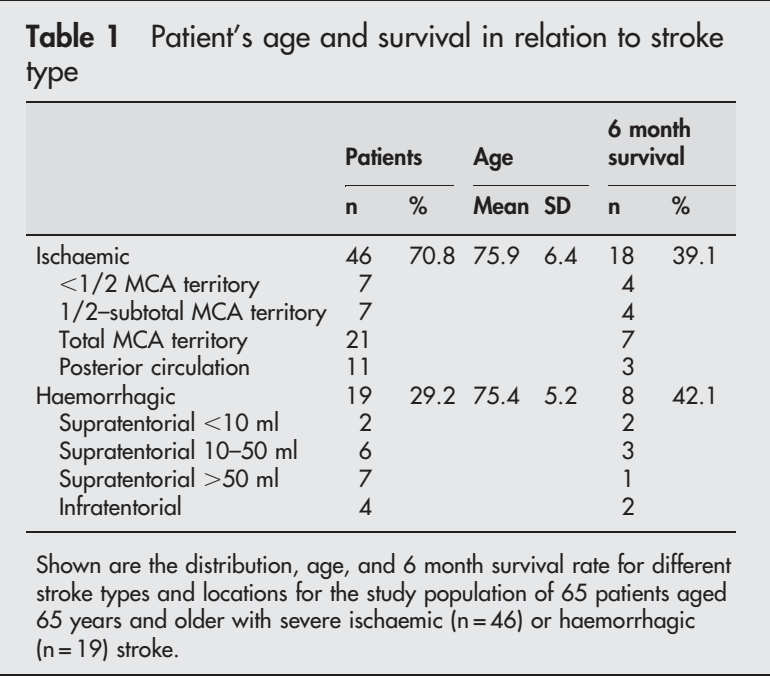




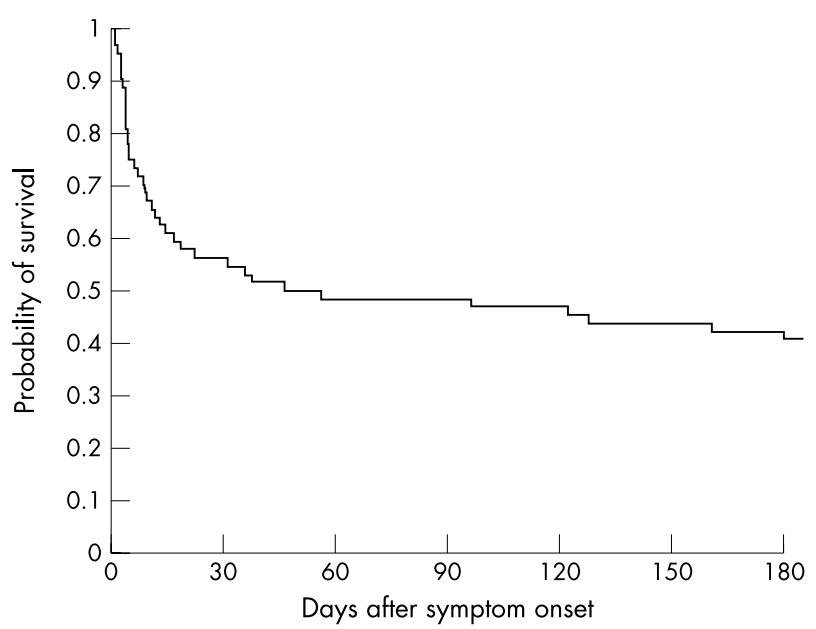

Figure 1 Kaplan-Meier survival curve of 65 mechanically ventilated stroke patients aged 65 years and older up to 6 months ( 180 days) following stroke. Note that the majority of deaths $(74 \%)$ occurred within the first 30 days following stroke.

From a total of 20 demographic, clinical, and laboratory variables tested in a univariate analysis ( $\chi^{2}$ test), we identified four variables that were positively or negatively correlated with survival at 6 months (table 2): negative predictors for survival were age $>77.5$ years, WBC $>10 / \mathrm{nl}$, and serum glucose $>10.8 \mathrm{mmol} / \mathrm{l}(180 \mathrm{mg} / \mathrm{dl})$ on admission, whereas elective intubation was the only significant positive predictor for survival. We found no significant differences in survival rates between different stroke subtypes (ischaemic $v$ haemorrhagic) or location (anterior $v$ posterior, supratentorial $v$ infratentorial, respectively).

In a second step, multiple logistic regression analysis was performed for the same 20 variables to obtain independent predictors of survival after 6 months which left the following three variables: age $>77.5$ years (negatively correlated with an OR of $0.14 ; 95 \%$ CI 0.04 to $0.53 ; \mathrm{p}=0.004$ ), white blood count $>10 / \mathrm{nl}$ (negatively correlated with an OR of $0.31 ; 95 \%$
CI 0.11 to $0.90 ; \mathrm{p}=0.032$ ), and elective intubation (positively correlated with an OR of 13.6; 95\% CI 2.7 to 68.6; $\mathrm{p}=0.002$ ).

\section{Results of the cross-sectional survey}

By the time of the cross-sectional survey, another two patients (one ischaemic, one haemorrhage) had died, resulting in a survival rate of $36.9 \%$ (24 out of 65 ). Two patients refused to take part in the cross-sectional survey, thus the following results refer to the remaining 22 patients.

The outcome as reflected by BI and mRS at the time of the cross-sectional survey is illustrated in fig 2. Outcome was poor in 14 patients $(\mathrm{BI}<35)$, moderate in three (BI 35-70) and good in five patients $(\mathrm{BI}>70)$. Outcome results from the mRS were similar with the majority of patients $(n=16)$ scoring 4-5 points reflecting high dependency, while three patients each scored $2-3$ or $0-1$ points. Ten patients lived at home, and 12 in a nursing home.

To meet the requirements of the definition of QoL as a measure of the "individual's perception", we present patient reports of the SA-SIP30 separately from the proxy reports. Eleven patients were able to complete the questionnaires on their own or with only minimal proxy assistance. This "selfrating" group revealed a median BI of 65 (25th, 75th percentile: 10, 95), and an average SA-SIP30 score of 48.0 (SD 27.3 ) \%. Mean scores were higher in the physical (61.8 $(39.8) \%$ ) than in the psychosocial domain (33.4 (26.5) \%). In this group eight out of 11 patients would again decide in favor of intensive care treatment, one would have decided against it, and another two were undecided.

The remaining 11 patients fell into the "proxy-rating" group with a median BI of 5 (25th, 75 th percentile: 0, 25), and an average SA-SIP30 score of 67.8 (17.2) \%. Again, mean scores were higher in the physical $(79.2(20.4) \%)$ than in the psychosocial domain (50.6 (30.6) \%). In this group, six proxies would in retrospect decide in favor of intensive care treatment, while three would have decided against and another two were undecided.

As is illustrated in fig 3, both the "self-rating" (A) and the "proxy-rating" (B) groups reported items from the physical domain such as household management, mobility, body care/ movement, and ambulation as most markedly impaired,

\begin{tabular}{|c|c|c|c|c|}
\hline Variable & n $(\%)$ & OR & $95 \% \mathrm{Cl}$ & p Value \\
\hline Male sex & $35(54)$ & 1.00 & $0.37-2.71$ & 1.00 \\
\hline Age $>77.5$ years & $22(34)$ & 0.14 & $0.04-0.53$ & $0.004^{*}$ \\
\hline Ischaemic stroke & 46 (71) & 0.88 & $0.30-2.62$ & 0.82 \\
\hline Posterior circulation/ infratentorial & $15(23)$ & 0.69 & $0.21-2.32$ & 0.55 \\
\hline Elective intubation & $13(20)$ & 13.6 & $2.68-68.6$ & $0.002^{*}$ \\
\hline \multicolumn{5}{|l|}{ Cardiovascular risk factors } \\
\hline Hypertension & $42(65)$ & 2.58 & $0.85-7.82$ & 0.10 \\
\hline Diabetes mellitus & $28(43)$ & 1.23 & $0.45-3.35$ & 0.68 \\
\hline Atrial fibrillation & $39(60)$ & 0.85 & $0.31-2.34$ & 0.76 \\
\hline Hypercholesterinemia & $11(17)$ & 0.51 & $0.12-2.12$ & 0.35 \\
\hline \multicolumn{5}{|l|}{ Neurological deficits on admission } \\
\hline Coma & $27(42)$ & 0.47 & $0.17-1.33$ & 0.15 \\
\hline Anisocoria & $18(28)$ & 0.48 & $0.15-1.55$ & 0.22 \\
\hline Absent unilateral pupillary light reflex & $4(6)$ & 0.48 & $0.05-4.89$ & 0.54 \\
\hline Absent bilateral pupillary light reflex & $9(14)$ & 0.38 & $0.07-2.00$ & 0.25 \\
\hline Absent bilateral corneal reflex & $8(12)$ & 0.18 & $0.02-1.59$ & 0.12 \\
\hline Unilateral Babinski's sign & $26(40)$ & 2.00 & $0.72-5.53$ & 0.18 \\
\hline Bilateral Babinski's sign & $15(23)$ & 0.29 & $0.07-1.17$ & 0.08 \\
\hline Forced gaze deviation & $32(49)$ & 1.36 & $0.50-3.68$ & 0.54 \\
\hline \multicolumn{5}{|l|}{ Laboratory analysis on admission } \\
\hline White blood count $>10 / \mathrm{nl}$ & $34(52)$ & 0.31 & $0.11-0.90$ & $0.032^{*}$ \\
\hline C-reactive protein $>3 \mathrm{mg} / \mathrm{dl}$ & $15(23)$ & 0.74 & $0.22-2.50$ & 0.62 \\
\hline Glucose $>180 \mathrm{mg} / \mathrm{dl}$ & $21(32)$ & 0.26 & $0.07-0.90$ & $0.034^{*}$ \\
\hline \multicolumn{5}{|c|}{$\begin{array}{l}\text { Univariate association of } 20 \text { variables and the probability of survival up to } 6 \text { months after symptom onset in } 65 \\
\text { mechanically ventilated elderly ( } 65 \text { years and older) stroke patients. } \mathrm{Cl} \text {, confidence interval; OR, odds ratio; } p \text { was } \\
\text { obtained using } \chi^{2} \text { test. }\end{array}$} \\
\hline
\end{tabular}



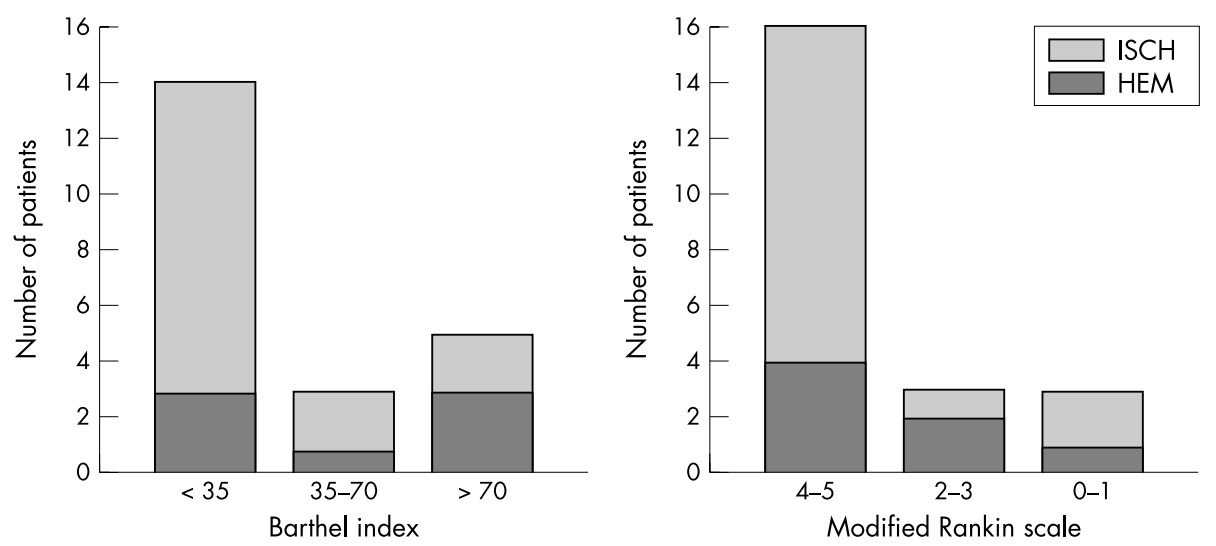

Figure 2 Outcome at the time of the cross-sectional survey as rated by Barthel Index (BI) and modified Rankin Scale (mRS). Patients with ischaemic stroke (ISCH; grey bars) are shown separately from those with haemorrhagic stroke (HEM; black bars).

whereas items from the psychosocial domain like emotional behavior, alertness behavior, social interaction, and communication were less severely affected.

\section{DISCUSSION}

The present study shows that the survival rate of mechanically ventilated elderly stroke patients ( $>65$ years) is comparable to mechanically ventilated stroke patients unselected for age. Our 6 month survival rate of $40 \%$ corresponds well to the 1 year survival rates of $39 \%$ and $33.1 \%$ recently reported by others in comparable groups of patients with ischaemic and haemorrhagic stroke, but unselected for age. ${ }^{34}$ This finding is remarkable, since it is known from earlier studies that older stroke patients have a poorer prognosis in general, ${ }^{17}{ }^{18}$ and age $>65$ years was identified as one of three independent predictors of 2 month mortality in mechanically ventilated stroke patients. ${ }^{4}$ In another study, age $>70$ years was associated with an odds ratio of 2.38 for death, but the finding did not reach significance. ${ }^{19}$ Two studies could, however, not identify age as an independent risk factor. ${ }^{3} 20$ These conflicting results are probably due to a number of other factors influencing mortality and outcome in critical care patients in general which may interact with the factor age, for example additional diseases such as heart disease, pulmonary disease, or diabetes. This assumption is in part supported by our finding that hyperglycaemia on admission
( $>10.8 \mathrm{mmol} / \mathrm{l})$ and elevated $\mathrm{WBC}$ were negative predictors for survival in a univariate factor analysis.

This study focused on survival and outcome of a subpopulation of stroke patients who were elderly and required $\mathrm{MV}$, that is, a combination of factors that could be expected to lead to a particularly poor outcome. However, elective intubation, as we defined it for the purpose of this study (see Methods), was the only positive predictor of survival. This finding is clinically highly relevant, because patients with severe stroke are often not cooperative enough to allow the diagnostic procedures needed for optimal treatment, for example, CT scan, MRI, or intra-arterial angiography. In practice, there may be second thoughts about intubating in such a situation just because the "patient is too old". In contrast, we found an odds ratio of 2.5 for 6 month survival of electively intubated patients, confirming the findings of Steiner and coworkers ${ }^{4}$ in a group of mechanically ventilated stroke patients unselected for age. We can exclude that the higher fatality in our non-electively intubated patients was due to differences in duration of MV which may have led to a higher incidence of ventilatorassociated complications, because the duration of MV in our sample (5-7 days on average) was well within the range previously published for stroke patients, ${ }^{319}$ and duration of MV was not significantly different between the three indications in our patients. This can be explained by the fact
A

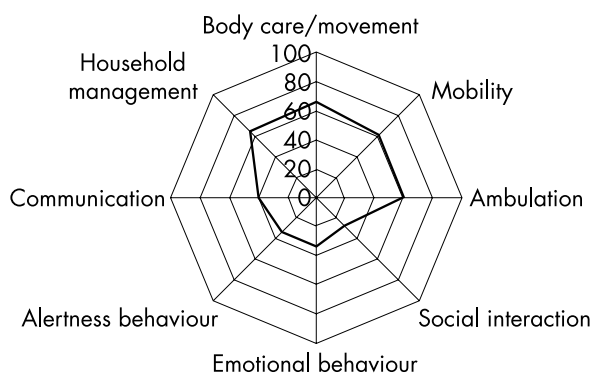

Emotional behaviour

SA-SIP 30 subscales

(mean \% of max. dysfunction)
B

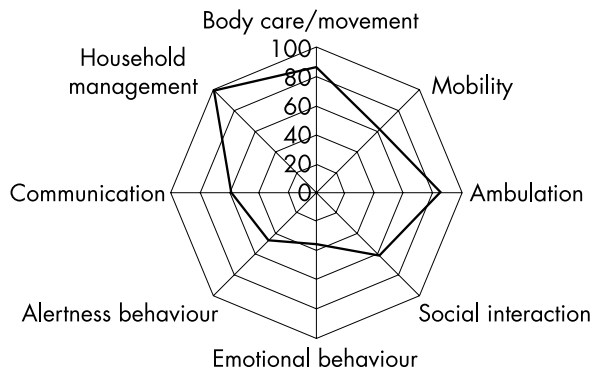

SA-SIP 30 subscales

(mean \% of max. dysfunction)

Figure 3 Results from the SA-SIP30 of 22 patients at the time of the cross-sectional analysis. The average dysfunction in the eight subscales (group mean percentages of maximum dysfunction) is shown in a cobweb diagram with $20 \%$ increments on the $y$ axis. (A) refers to 11 patients able to complete the questionnaire on their own, whereas (B) shows the group of 11 patients rated by proxies. The data were adjusted for different subgroup sizes. 
that in our terms, "elective intubation" only referred to the initial indication, for example for imaging procedures. In a number of cases however, a large ischaemic or haemorrhagic stroke was revealed, requiring prolonged MV for neurological reasons (for example, hyperventilation in the context of antioedematous therapy). Thus, our results may imply that elective intubation and ventilation to allow certain diagnostic procedures may have favourably influenced the clinical course.

We also identified a critical age, beyond which prognosis for survival is extremely poor in mechanically ventilated stroke patients independent of other factors. We used the current average life expectancy at birth for Germany ( 77.5 years) for dichotomisation, and our data correspond well with the finding that the combination of age $>80$ years and MV longer than 3 days is associated with a very poor prognosis in a general ICU population. ${ }^{21}$

In the present study, functional outcome was worse compared to results from the literature. Using the BI as the primary measure for functional outcome, we found that only eight out of 22 patients (36\%) recovered to a $\mathrm{BI} \geqslant 60$ points (defined in the literature as moderate to good functional outcome), in contrast to the previously published approximately $60 \%$ in patients unselected for age. ${ }^{3}{ }^{4}$ However, it is noteworthy that, out of those eight patients with a $\mathrm{BI} \geqslant 60$, five reached a $\mathrm{BI} \geqslant 90$, equivalent to a favourable functional status. It should also be stressed, that of those 25 patients older than 77.5 years, only one survived up to the crosssectional survey with a disastrous functional outcome. We therefore conclude that MV in stroke patients around or beyond the age of 80 should only be considered in exceptional situations, for example, when elective intubation for an invasive procedure (such as ventricular drainage) is needed and MV can forseeably be limited to a few hours.

Examiner ratings on functional outcome such as BI or mRS have been criticised for not necessarily reflecting subjective QoL, ${ }^{22}$ but to our knowledge this issue has not been addressed in previous studies on outcome in mechanically ventilated stroke patients. As an additional measurement for outcome, we therefore asked patients to assess their QoL in a crosssectional survey using a validated stroke-specific QoL scale (SA-SIP30). This scale provides additional information on the mainly affected aspects of QoL. In a previous study, the mean SA-SIP30 in a large group of consecutive unselected stroke patients with a mean age of 70 (SD 3) years was found to be $32.0(20.7) \%$ at 6 months following stroke. ${ }^{23}$ In contrast, our group of patients was clearly more severely affected (mean SA-SIP $48.0(27.3) \%$ and 67.8 (17.2) \% for the "self-rating" and the "proxy-rating" group, respectively) in both the physical and the psychosocial subcategories. In general, motor functions (household management, body care, ambulation) were more impaired, whereas psychosocial functions (alertness, communication, emotional behaviour) were less affected.

It should be noted that our study is subject to some limitations. First, the study design was observational rather than an intention to treat protocol. Since no data on the number of cases in whom patients, proxies, or physicians decided against intensive care treatment were available, a selection bias has to be assumed. Second, due to the relatively small sample size, no significant effects were obtained for a number of factors (such as certain neurological deficits) potentially influencing outcome and QoL. Third, QoL was only assessed in those patients who survived up to the time of the cross-sectional survey, which may lead to an overestimation of QoL. On the other hand, only two patients died between the 6 month follow up and the cross-sectional survey, and in stroke patients in general there is a trend for further recovery of function for up to 2 years following stroke so that QoL could also be underestimated.
Our data show that approximately one in four elderly patients suffering a severe stroke requiring intubation and $\mathrm{MV}$, and surviving the first 6 months may recover to a largely independent functional status with moderate to good QoL ratings. Due to a possible selection bias, however, this result cannot easily be extrapolated to a general elderly population. Whether in the strict medico-economic sense the administration of intensive care treatment and MV in elderly stroke patients is cost effective remains undetermined. One recent study on this topic concluded that it was cost effective for extending life, but not for preserving QoL, ${ }^{8}$ but outcome in that population was markedly worse $(<10 \%$ of 1 year survivors reached functional independence) compared to the results of other studies ${ }^{13424}$ and compared to our own results.

We conclude that the age limit up to which MV in stroke patients appears to be warranted is around 80 years, that is, in the same range as in general medical patients receiving intensive care therapy. Notwithstanding the fact that approximately $60 \%$ of patients intubated in the acute phase of stroke die within the first 6 months, we could show that still one in four survivors can be expected to recover to a good functional outcome with a reasonable quality of life.

\section{ACKNOWLEDGEMENTS}

We are indebted to Mary Gossen ( $\mathrm{PhD}$ ) for language editing. We thank all patients and their relatives for their participation and kind cooperation in this study.

\section{Authors' affiliations}

C Foerch, K R Kessler, D A Steckel, H Steinmetz, M Sitzer, Department of Neurology, Johann Wolfgang Goethe-University, Frankfurt am Main, Germany

Competing interests: none declared

\section{REFERENCES}

1 Wijdicks EF, Scott JP. Causes and outcome of mechanical ventilation in patients with hemispheric ischemic stroke. Mayo Clin Proc 1997;72(3):210-3.

2 Guijar AR, Deibert E, Manno EM, et al. Mechanical ventilation for ischemic stroke and intracerebral hemorrhage: indications, timing, and outcome. Neurology 1998:51(2):447-51.

3 Bushnell CD, Phillips-Bute BG, Laskowitz DT, et al. Survival and outcome after endotracheal intubation for acute stroke. Neurology 1999;52(7):1374-81.

4 Steiner T, Mendoza G, De Georgia M, et al. Prognosis of stroke patients requiring mechanical ventilation in a neurological critical care unit. Stroke 1997;28(4):711-5.

5 Wolfe CD, Rudd AG, Howard, et al. Incidence and case fatality rates of stroke subtypes in a multiethnic population. The South London Stroke Register. J Neurol Neurosurg Psychiatry 2002;72:211-6.

6 Hollander M, Koudstaal PJ, Bots ML, et al. Incidence, risk, and case fatality of first ever stroke in the elderly population. The Rotterdam Study. J Neurol Neurosurg Psychiatry 2003;74:317-21.

7 Grotta J, Pasteur W, Khwaja G, et al. Elective intubation for neurologic deterioration after stroke. Neurology 1995;45(4):640-4.

8 Mayer SA, Copeland D, Bernardini GL, et al. Cost and outcome of mechanical ventilation for life-threatening stroke. Stroke 2000;31(10):2346-53.

9 Arbeitsgruppe-Schlaganfall-Hessen. Schlaganfallversorgung in der Akutphase. Erste Auswertung eines Datenbank fuer die begleitende externe Qualitaetssicherung in Hessen. Akt Neurologie 1999;26:8-16.

10 Dobkin B. Management of the patient with stroke. Neurology 1995;45(suppl 1):S1-35.

11 Kessler S, Jaeckel W, Cziske R. Assessing health in musculoskeletal disorders - the appropriateness of a German version of the Sickness Impact Profile. Rheumatol Int 1997;17(3):119-25.

12 Mahoney FI, Barthel DW. Functional evaluation: the Barthel Index. Md State Med J 1965;14:61-5.

13 van Swieten JC, Koudstaal PJ, Visser MC, et al. Interobserver agreement for the assessment of handicap in stroke patients. Stroke 1988;19(5):604-7.

14 Bergner M, Bobitt RA, Carter WB, et al. The Sickness Impact Profile: development and final revision of a health status measure. Med Care $1981 ; 19: 787-805$

15 van Straten A, de Haan RJ, Limburg M, et al. A stroke-adapted 30-item version of the Sickness Impact Profile to assess quality of life (SA-SIP30). Stroke 1997;28(11):2155-61.

16 Statistisches Bundesamt. Weitere Zunahme der Lebenserwartung. Available online at: http://www.destatis.de/basis/d/bevoe/bevoetab3.htm (accessed Nov 14, 2002) 
17 Howard G, Walker MD, Becker C, et al. Community hospital-based stroke programs: North Carolina, Oregon, and New York. III. Factors influencing survival after stroke: proportional hazards analysis of 4219 patients. Stroke 1986;17(2):294-9.

18 Bonita R, Ford MA, Stewart AW. Predicting survival after stroke: a three-year follow-up. Stroke 1988;19(6):669-73.

19 Berrouschot J, Rossler A, Köster J, et al. Mechanical ventilation in patients with hemispheric ischemic stroke. Crit Care Med 2000;28(8):2956-61.

20 Rordorf G, Koroshetz W, Efird JT, et al. Predictors of mortality in stroke patients admitted to an intensive care unit. Crit Care Med 2000;28(5):1301-5
21 Cohen IL, Lambrinos J, Fein IA. Mechanical ventilation for the elderly patient in intensive care. Incremental changes and benefits. JAMA 1993;269(8): 1025-9

22 Buck D, Jacoby A, Massey A, et al. Evaluation of measures used to assess quality of life after stroke. Stroke 2000;31(8):2004-10.

23 van Straten A, de Haan RJ, Limburg $M$, et al. Clinical meaning of the StrokeAdapted Sickness Impact Profile-30 and the Sickness Impact Profile-136. Stroke 2000;31(11):2610-5.

24 Burtin $\mathrm{P}$, Bollaert PE, Feldmann L, et al. Prognosis of stroke patients undergoing mechanical ventilation. Intensive Care Med 1994;20(1):32-6

\section{NEUROLOGICAL PICTURE}

\section{Triple carotid aneurysms in a patient with migraine attacks}

A 28 year old woman presented with a history of worsening migraine headaches for 15 years. She reported to have right temporal headaches several times a month accompanied by nausea and photophobia. The patient was treated with flunarizine and rizatriptan $10 \mathrm{mg}$ daily with little response.

MRI of the brain showed findings suggestive of a vascular lesion adjacent to the right cavernous sinus. Digital subtraction angiography (DSA) revealed three aneurysms in the petrous and cavernous segments of the right internal carotid artery unusually situated like "pearls on a string" (fig 1). The most distally located aneurysm arose from the C5 portion, measured $18 \mathrm{~mm}$ in diameter and caused a mass effect within the posterior cavernous sinus region. Three-dimensional images obtained after angiography clearly revealed a complex anatomy consisting of rather fusiform dilatations without clear distinction between the neck of the aneurysms and the parent artery (fig 2). Permanent carotid occlusion was performed successfully and she recovered from her migraine episodes with only occasional minor headaches remaining.

Triple carotid aneurysms as described here are extremely uncommon and represent a therapeutic challenge, particularly if located at the skull base. The association of a carotid

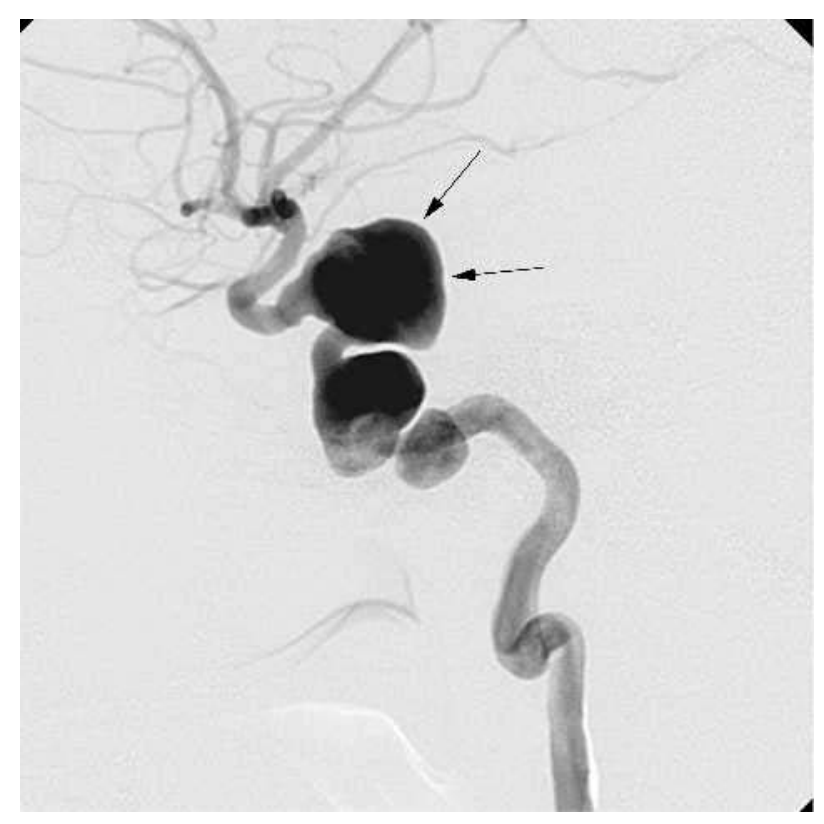

Figure 1 DSA of the right internal carotid artery. Lateral view reveals three aneurysms involving the petrous and the cavernous segment. The most distal one, arising from the C5 portion, has a diameter of $18 \mathrm{~mm}$ and is directed cranially and posteriorly (arrows). aneurysm and migraine like headaches in older patients has been described in only a few case reports. ${ }^{12}$ Patients with longstanding headaches or migraine should undergo a brain imaging to exclude an underlying vascular cause which may allow curative treatment. High quality 3D imaging of vascular structures significantly improves understanding of complex vascular anatomy and may facilitate clinical decision making.

\section{G Benndorf}

R M Naeini

Department of Radiology, Baylor College of Medicine, Houston, Texas, USA

T N Lehmann

Department of Neurosurgery, Charite, Humboldt University, Berlin, Germany

Correspondence to: Dr G Benndorf, Department of Radiology, Baylor College of Medicine, One Baylor Plaza, MS 360, Houston, Texas 770303498, US; benndorf@bcm.tmc.edu

\section{References}

1 Alberti A, Sarchielli P, Mazzotta G, et al. Migraine-like headache in a patient with a giant aneurysm of the intracranial internal carotid artery. Cephalalgia 2001;21(4):511.

2 Narbone MC, Rao R, Grugno R, et al. A late 'migraine': the only symptom of an intrasellar aneurysm. Headache 1997;37(8):527-8.

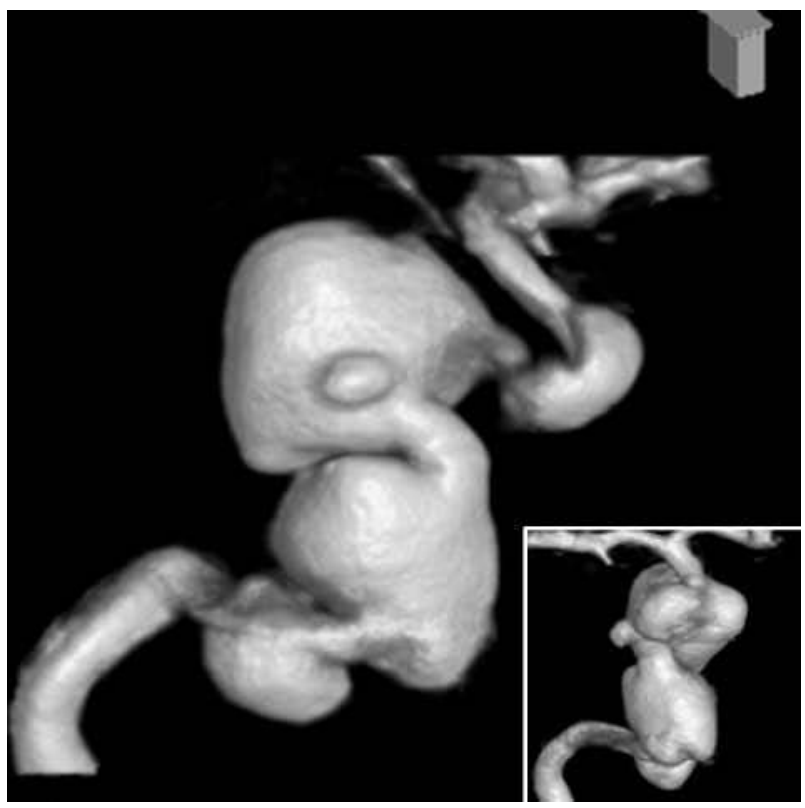

Figure $23 D$ image (3D DSA, surface shaded display) showing the medial aspect of the aneurysms. Only the small proximal aneurysm has a saccular shape. The two larger aneurysms appear to be fusiform type dilatations without discrete separation between aneurysmal neck and parent vessel (miniature: A-P view) 